DESAFIOS: Revista Interdisciplinar da Universidade Federal do Tocantins - V. 2 - n. 02. p.283-306, jan/jun. 2016.

DOl: http://dx.doi.org/10.20873/uft.2359-3652.2016v2n2p283

\title{
PERFIL CLÍNICO-EPIDEMIOLÓGICO DE MULHERES COM NEOPLASIA DE MAMA ATENDIDAS NO HOSPITAL REGIONAL DE REFERÊNCIA NO MUNICÍPIO DE ARAGUAÍNA-TO NO PERÍODO DE 2000 A 2015
}

\author{
CLINICAL-EPIDEMIOLOGICAL PROFILE OF WOMEN WITH BREAST CANCER \\ TREATED AT THE REGIONAL HOSPITAL OF REFERENCE IN THE MUNICIPALITY OF \\ ARAGUAÍNA- TO IN THE PERIOD 2000 TO 2015
}

Maianna Macêdo de Sousa Sabrina Barreto Figueredo

Reinaldo Magalhães Fernandes

Instituto Tocantinense Presidente Antônio Carlos - FAHESA/ITPAC

\section{RESUMO}

O câncer de mama é uma neoplasia rara antes dos 35 anos de idade, acometendo em cerca de 99\% as mulheres. Ainda possui etiologia desconhecida, no entanto apresenta alguns fatores de risco importantes, tais como: menarca precoce antes dos 12 anos, menopausa tardia acima de 50 anos, primeira gestação acima de 35 anos, nuliparidade, idade, fatores genéticos, além de fatores relacionados à estilo de vida como consumo de tabaco e histórico de consumo de bebidas alcoólicas. No Brasil, o câncer de mama também é o mais incidente (excluídos os tumores de pele não melanoma) em mulheres de todas as regiões, exceto na região Norte, onde o câncer do colo do útero ocupa a primeira posição. De acordo com a Organização Mundial de Saúde (OMS) como ação de prevenção secundária, ou seja, de detecção precoce do câncer de mama, são mencionadas três estratégias complementares entre si, que são: o auto-exame das mamas, o exame clínico e a mamografia, sendo que tais fatores podem ser influenciados pelo nível de escolaridade das mulheres. É incomum a associação com fatores hereditários, sendo de suma importância a detecção e tratamento precoces adequados de forma a contribuir para um prognóstico favorável. Objetivo: Descrever o perfil clínicoepidemiológico das Neoplasias de Mama em pacientes do sexo feminino atendidas no Hospital Regional de Referência de Araguaína-TO, no período de 2000-2015. Metodologia: Quanto à natureza o estudo pode ser classificado como quantitativo, pois se baseará na geração de dados e na análise crítica dos mesmos. Será de caráter descritivo, retrospectivo e documental realizado no Hospital Regional de Referência de Araguaína através de informações que serão coletadas por meio da análise de fichas do Registro Hospitalar do Câncer disponibilizadas pelo Instituto Nacional do Câncer (INCA).

Palavras-chave: Câncer de Mama - perfil clínico-epidemiológico e fatores de risco

\begin{abstract}
Breast cancer is a rare neoplasm before 35 years of age, affecting approximately $99 \%$ women. Still has unknown etiology, however presents some important risk factors, such as: early menarche before 12 years, late menopause above 50 years, first pregnancy above 35 years, nulliparity, age, genetic factors, in addition to factors related to lifestyle as the consumption of tobacco and history of consumption of alcoholic beverages. In Brazil, breast cancer is also the most incident (excluding non-melanoma skin tumors) in women of all regions, except in the northern region, where the cervical cancer occupies the first position. According to the World
\end{abstract}


Health Organization (WHO) as secondary prevention action, i.e. for early detection of breast cancer, are mentioned three complementary strategies among themselves, which are: the breast self-exam, the clinical examination and mammography, being that such factors may be influenced by the level of education of women. It is uncommon to association with hereditary factors and highlighted the importance of early detection and treatment appropriate to contribute to a favorable prognosis. Objective: To describe the clinical-epidemiological profile of Breast Neoplasms in female patients answered the Regional Hospital of reference in the municipality of Araguaína- TO, in the period 2000-2015. Methodology: As to the nature of the study can be classified as quantitative, because it is based on data generation and critical analysis of the same. Will be a descriptive, retrospective and documentary checks carried out at the Regional Hospital of reference in the municipality of Araguaína- TO through the information that will be collected by means of the analysis of the connectors of the Cancer Hospital Registry made available by the National Cancer Institute.

Keywords: Breast Cancer - clinical- epidemiological profile and risk factors

Recebido em 25/05/2016. Aceito em 30/05/2016. Publicado em 31/05/2016.

\title{
INTRODUÇÃO
}

\begin{abstract}
Neoplasia significa "novo crescimento", e um novo crescimento é denominado neoplasma. O termo tumor foi originalmente aplicado ao edema causado por inflamação, mas o uso não neoplásico de tumor praticamente desapareceu; portanto o temos atualmente se iguala a neoplasma (LESTER, 2010, p.260). [...] Os tumores malignos são referidos coletivamente como cânceres, um derivado da palavra latina caranguejo, pois se aderem a qualquer região em que estejam, de maneira obstinada, similar a um caranguejo (LESTER, 2010).
\end{abstract}

Antes dos 35 anos de idade o câncer de mama é incomum, aproximadamente $4 \%$ a 5\% dos casos, crescendo rápida e progressivamente acima desta faixa etária, tendo pico entre 45 e 55 anos. O câncer de mama em homens é raro e corresponde em torno de $1 \%$ dos casos (ANDRADE, 2014).

O câncer de mama ainda tem etiologia desconhecida, no entanto apresenta alguns fatores de risco tais como: menarca precoce (antes dos 12 anos), menopausa tardia (acima de 55 anos), primeira gestação acima de 30 anos, nuliparidade, idade, fatores genéticos, ingestão de bebidas alcoólicas, sobrepeso, obesidade, tabagismo (INCA, 2015).

O controle do câncer de mama é fundamentalmente baseado na prevenção, na detecção precoce e no tratamento. Vários estudos relatam sobre o impacto de mudanças nos padrões comportamentais da infância à idade adulta, e tem sugerido que as intervenções desde as fases iniciais da vida podem ser mais eficazes do que as ações isoladas de prevenção, 
DESAFIOS: Revista Interdisciplinar da Universidade Federal do Tocantins - V. 2 - n. 02. p.283-306, jan/jun. 2016.

DOl: http://dx.doi.org/10.20873/uft.2359-3652.2016v2n2p283

tratamento e cura, quando consideradas a incidência e a mortalidade por câncer (ANDRADE, 2014).

De acordo com a Organização Mundial de Saúde (OMS) como ação de prevenção secundária, ou seja, de detecção precoce do câncer de mama, são mencionadas três estratégias complementares entre si, que são: o auto-exame das mamas, o exame clínico e a mamografia (INCA, 2015).

\section{OBJETIVOS}

\section{Objetivo geral}

Descrever o perfil clínico-epidemiológico das Neoplasias de Mama em pacientes do sexo feminino atendidas no Hospital Regional de Referência de Araguaína-TO, no período de 2000-2015.

\section{Objetivos específicos}

1) Verificar retrospectivamente através de levantamento de dados a faixa etária, cor, escolaridade, estado civil, tabagismo e etilismo em pacientes com neoplasia de mama;

2) Analisar retrospectivamente através de levantamento de dados o tipo histológico, estadiamento da doença e hereditariedade das pacientes com neoplasia de mama;

3) Relacionar o grau de estadiamento com faixa etária, tipo histológico com faixa etária e estadiamento com ano do diagnóstico.

\section{JUSTIFICATIVA}

No Brasil, o câncer de mama devido a sua alta incidência e mortalidade representa um importante problema de saúde pública e tem justificado a realização de várias pesquisas sobre sua epidemiologia, fatores de risco, etiologia, métodos diagnósticos, prevenção e tratamento (RODRIGUES, 2010).

A assistência ao tratamento oncológico está entre as mais caras no âmbito social, por incluir custos diretos como exames preventivos, testes diagnósticos e tratamentos, e custos indiretos consequentes da incapacidade produtiva do doente e morbi-mortalidade relacionada ao câncer e/ou tratamento (RODRIGUES, 2010). 
Para mudança do cenário atual do câncer de mama, é necessário que haja estímulo à busca de informações qualificadas sobre a incidência e prevalência da doença nas populações, propiciando a implantação de politicas públicas que levem a realização de ações efetivas de prevenção e detecção precoce, objetivando a redução de danos, as taxas de mortalidade e as despesas públicas. Nesse sentido, este estudo tem por objetivo caracterizar o perfil clínico epidemiológico das pacientes atendidas no ambulatório de Especialidades Médicas do HRA.

\section{MATERIAL E MÉTODOS}

\section{ASPECTOS ÉTICOS DA PESQUISA}

Trata-se de um estudo retrospectivo com análise de informações adquiridos de uma base de dados públicos do Registro Hospitalar de Câncer (RHC) https://irhc.inca.gov.br/RHCNet/, não lidando diretamente com o paciente, com isso os riscos aos indivíduos da amostra são mínimos. Os pesquisadores comprometem-se a preservar o anonimato dos sujeitos da pesquisa, minimizando os riscos.

Considerando que a pesquisa é de caráter retrospectivo utilizando dados de fonte de domínio público do Registro Hospitalar de Câncer (RHC) https://irhc.inca.gov.br/RHCNet/, foi solicitado a dispensa da utilização do Termo de Consentimento Livre e Esclarecido (TCLE) para todos os sujeitos da pesquisa.

O Termo de Anuência da Instituição não se aplica uma vez que a coleta de informações será em base de dados de domínio público.

\section{TIPO DE ESTUDO}

Quanto à natureza o estudo pode ser classificado como quantitativo, pois se baseia na geração de dados e na análise crítica dos mesmos. É de caráter descritivo, retrospectivo e documental realizado no Hospital Regional de Referência de Araguaína através de informações que foram coletadas por meio da análise de fichas do Registro Hospitalar do Câncer disponibilizadas pelo Instituto Nacional do Câncer (INCA).

\section{LOCAL DA PESQUISA E UNIVERSO (SUJEITOS)}

Local- Base de dados do INCA/ RHC - Registro Hospitalar de Câncer https://irhc.inca.gov.br/RHCNet/. Sujeitos- pacientes diagnosticadas com neoplasia de mama no período de 2000 a 2015 em Araguaína (TO). 


\section{AMOSTRA E CASUÍSTICA}

A população estudada é composta por pacientes com neoplasia de mama atendidos no HRRA/TO no período de 2000-2015. As identificações das neoplasias foram realizadas na base de dados do RHC (Registro Hospitalar de Câncer), para obtenção das principais informações documentadas sobre os pacientes e a doença.

Foram excluídos os casos de neoplasia de mama no sexo masculino e de pacientes tratados anteriormente e posteriormente ao período do estudo. Como critério de inclusão na casuística se estabeleceu: diagnóstico histopatológico de câncer de mama no sexo feminino matriculadas e registrado no RHC.

As variáveis analisadas foram: faixa etária, cor, escolaridade, estado civil, tabagismo, etilismo, tipo histológico, estadiamento da doença e hereditariedade.

\section{COLETA E PROCESSAMENTO DE DADOS}

O levantamento de dados clínicos e epidemiológicos dos pacientes foram revistos/computados/coletados através da coleta de informações em base de dados de domínio público - Registro Hospitalar de Câncer (RHC) https://irhc.inca.gov.br/RHCNet/.

\section{ANÁLISE ESTATÍSTICA}

O material gráfico resultante da etapa de tabulação foram apresentados em tabelas com as frequências absolutas e relativas para variáveis de interesse assim como o cruzamento entre elas.

\section{ANÁLISE CRÍTICA DOS RISCOS E BENEFÍCIOS}

Trata-se de um estudo retrospectivo com análise de dados clínicos e epidemiológicos de pacientes através da coleta de informações em base de dados de domínio público (Registro Hospitalar do Câncer), não lidando diretamente com o paciente, com isso os riscos aos indivíduos da amostra são mínimos. Os pesquisadores comprometem-se a preservar o anonimato dos sujeitos da pesquisa, minimizando os riscos.

O estudo apresenta como benefícios: o conhecimento - em âmbito local - do perfil clínico-epidemiológico das pacientes com diagnóstico de Câncer de Mama, considerando que Araguaína atende cidades circunvizinhas, tentando associá-los com as condições 
socioeconômicas, tipo histológico prevalente e fatores de risco encontrados; divulgação dos achados no meio científico.

\section{RESULTADOS}

Foram documentados 1146 casos de Neoplasia de mama no Registro Hospitalar de câncer (RHC) do Hospital Regional de Referência de Araguaína no período referente à pesquisa (2000 a 2015).

A idade de acometimento foi variada, com casos a partir de 19 anos e até acima dos 85 anos sendo observada maior prevalência na faixa etária de 45-49 anos, com 16,14\% ( $\mathrm{n}=185$ ), seguida de pacientes com 50-54 anos representando $14,74 \%$ da amostra $(n=169)$ e com 40-44 anos representando $13,08 \%$ da amostra $(n=150)$. A maioria das pacientes era da cor parda (839 pacientes representando 73,21\%), seguida de brancos (221 pacientes representando $19,28 \%$ ), negros (59 pacientes, representando 5,14\%) e amarelos (4 pacientes representando 0,34 pacientes). Do total de pacientes (1146) não foi obtido informação da cor de 23 pacientes, representando $2 \%$ do total.

Em relação à escolaridade 502 pacientes apresentavam o ensino fundamental incompleto, representando 43,80\% do total, 176 apresentavam o nível médio, representando $15,35 \%, 145$ pessoas não possuíam escolaridade, representando 12,73\%, 114 possuíam ensino fundamental completo, representando 9,94\%, 73 apresentavam nível superior, representando $6,36 \%$ e 6 possuíam nível superior incompleto, representando 0,52\%. Não foi obtida informação sobre escolaridade de 130 pacientes representando 11,34 \%. 
DESAFIOS: Revista Interdisciplinar da Universidade Federal do Tocantins - V. 2 - n. 02. p.283-306, jan/jun. 2016. DOI: http://dx.doi.org/10.20873/uft.2359-3652.2016v2n2p283

Tabela 1- Distribuição dos 1146 casos registrados no RHC do HRRA-TO de pacientes com neoplasia de mama conforme faixa etária, cor e grau de instrução durante período de 2000 a 2015.

Table 1 - Distribution of 1146 cases recorded in the RHC HRRA-TO of patients with breast cancer according to age, color and level of education during the period from 2000 to 2015.

\begin{tabular}{|c|c|c|}
\hline VARIÁVEIS & $\mathbf{N}$ & $\%$ \\
\hline \multicolumn{3}{|l|}{ FAIXA ETÁRIA } \\
\hline $15-19$ & 2 & 0,17 \\
\hline $20-24$ & 4 & 0,34 \\
\hline $25-29$ & 23 & 2 \\
\hline $30-34$ & 44 & 3,83 \\
\hline $35-39$ & 96 & 8,37 \\
\hline $40-44$ & 150 & 13,08 \\
\hline $45-49$ & 185 & 16,14 \\
\hline $50-54$ & 169 & 14,74 \\
\hline $55-59$ & 126 & 10,99 \\
\hline $60-64$ & 125 & 10,90 \\
\hline $65-69$ & 90 & 7,85 \\
\hline $70-74$ & 59 & 5,14 \\
\hline $75-79$ & 43 & 3,75 \\
\hline $80-84$ & 21 & 1,83 \\
\hline$>85$ & 9 & 0,78 \\
\hline \multicolumn{3}{|l|}{ RAÇA/COR } \\
\hline Parda & 839 & 73,21 \\
\hline Branca & 221 & 19,28 \\
\hline Preta & 59 & 5,14 \\
\hline Amarela & 04 & 034 \\
\hline Sem informação & 23 & 2 \\
\hline \multicolumn{3}{|l|}{ ESCOLARIDADE } \\
\hline $\begin{array}{l}\text { Fundamental } \\
\text { incompleto }\end{array}$ & 502 & 43,80 \\
\hline $\begin{array}{l}\text { Fundamental } \\
\text { completo }\end{array}$ & 114 & 9,94 \\
\hline Nível médio & 176 & 15,35 \\
\hline $\begin{array}{l}\text { Nível superior } \\
\text { incompleto }\end{array}$ & 6 & 0,52 \\
\hline $\begin{array}{l}\text { Nível superior } \\
\text { completo }\end{array}$ & 73 & 6,36 \\
\hline Nenhum & 145 & 12,73 \\
\hline Sem informação & 130 & 11,34 \\
\hline
\end{tabular}


Com relação ao estado conjugal, verificou-se que 56,98\% $(n=653)$ das pacientes eram casadas, $21,11 \%(n=242)$ eram solteiras, 14,39\% $(n=165)$ eram viúvas, 4,01\% $(n=46)$ eram separadas, 0,69 $(\mathrm{n}=8)$ apresentavam união consensual.

Quanto ao hábito de fumar foi verificado em 408 pacientes ( $\mathrm{n}=315$ consumidores e n=93 ex-consumidores). Em relação ao consumo de bebida verificou-se em 251 pacientes ( $\mathrm{n}=$ 218 consumidores e $n=33$ ex- consumidores).

Tabela 2 - Distribuição dos 1146 casos registrados no RHC do HRRA-TO de pacientes com neoplasia de mama conforme estado conjugal, histórico de consumo de bebida e histórico de consumo de tabaco durante período de 2000 a 2015.

Table 2 - Distribution of the 1146 cases recorded in the RHC HRRA-TO of patients with breast cancer as marital status, beverage consumption history and smoking history during the period 2000-2015.

\begin{tabular}{|c|c|c|}
\hline VARIÁVEIS & $\mathbf{N}$ & $\%$ \\
\hline \multicolumn{3}{|l|}{ ESTADO CONJUGAL } \\
\hline Casado & 653 & 56,98 \\
\hline Solteiro & 242 & 21,11 \\
\hline Viúvo & 165 & 14,39 \\
\hline Separado judicialmente & 46 & 4,01 \\
\hline União consensual & 8 & 0,69 \\
\hline Sem informação & 32 & 2,79 \\
\hline \multicolumn{3}{|l|}{$\begin{array}{l}\text { HISTÓRICO DE } \\
\text { CONSUMO DE BEBIDA }\end{array}$} \\
\hline Nunca & 769 & 67,10 \\
\hline Sim & 218 & 19,02 \\
\hline Ex-consumidor & 33 & 2,8 \\
\hline Sem informação & 126 & 10,99 \\
\hline \multicolumn{3}{|l|}{$\begin{array}{l}\text { HISTÓRICO DE } \\
\text { CONSUMO DE } \\
\text { TABACO }\end{array}$} \\
\hline Nunca & 620 & 54,10 \\
\hline Sim & 315 & 27,48 \\
\hline Ex-consumidor & 93 & 8,11 \\
\hline Sem informação & 118 & 10,29 \\
\hline
\end{tabular}

Em relação ao tipo histológico foram registrados 87,95\% (n=1008) das pacientes com o tipo carcinoma ductal infiltrante, 2,26\% $(\mathrm{n}=26)$ com carcinoma lobular, $0,87 \%(\mathrm{n}=10)$ carcinoma intraductal não infiltrante, ficando 8,90\% (n=102) com outros tipos histológicos.

Quanto ao estadiamento percebeu-se que a maioria das mulheres estudadas encontrava-se no estadiamento IIB, correspondendo a 17,88 $(n=205)$ e IIIA correspondendo a $17,80 \%(\mathrm{n}=204)$. 
Verificou-se que 45,20\% ( $\mathrm{n}=518)$ das pacientes apresentavam história familiar de neoplasia, 40,40\% (n=463) não apresentavam a mesma história e não foi obtida informação a respeito desse dado em $14,39 \%(n=165)$ das pacientes.

Tabela 3-Distribuição dos 1146 casos registrados no RHC do HRRA-TO de pacientes com neoplasia de mama conforme tipo histológico, estadiamento e história familiar de neoplasia durante período de 2000 a 2015.

Table 3-Distribution of 1146 cases recorded in the RHC HRRA-TO of patients with breast cancer as histologic type, stage and family history of cancer during the period 2000 to 2015.

\begin{tabular}{|c|c|c|}
\hline $\begin{array}{c}\text { VARIÁVEIS } \\
\text { TIPO HISTOIÓCICO }\end{array}$ & $\mathbf{N}$ & $\%$ \\
\hline Carcinoma ductal infiltrante & 1008 & 87,95 \\
\hline Carcinoma lobular & 26 & 2,26 \\
\hline $\begin{array}{l}\text { Carcinoma intraductal não } \\
\text { infiltrante }\end{array}$ & 10 & 0,87 \\
\hline Adenocarcinoma mucinoso & 9 & 0,78 \\
\hline Carcinoma papilífero & 11 & 0,95 \\
\hline Carcinoma ductal in situ & 5 & 0,43 \\
\hline $\begin{array}{l}\text { Outros carcinomas } \\
{ }^{1} \\
\text { Outros não carcinomas }\end{array}$ & $\begin{array}{l}61 \\
16\end{array}$ & $\begin{array}{l}5,32 \\
1,39\end{array}$ \\
\hline ESTADIAMENTO & $\mathbf{N}$ & $\%$ \\
\hline $\mathbf{0}$ & 14 & 1,22 \\
\hline I & 127 & 11,08 \\
\hline II A & 187 & 16,31 \\
\hline II B & 205 & 17,88 \\
\hline III A & 204 & 17,80 \\
\hline III B & 138 & 12,04 \\
\hline III C & 71 & 6,19 \\
\hline IV & 159 & 13,87 \\
\hline Sem Informação & 41 & 3,57 \\
\hline $\begin{array}{l}\text { HISTÓRIA FAMILIAR DE } \\
\text { NEOPLASIA }\end{array}$ & $\mathbf{N}$ & $\%$ \\
\hline Sim & 518 & 45,20 \\
\hline Não & 463 & 40,40 \\
\hline Sem informação & 165 & 14,39 \\
\hline
\end{tabular}

1 No grupo Outros carcinomas, encontra-se os tipos histológicos: Carcinoma Escamocelular, Carcinoma Adenoide Cístico, Carcinoma Medular, Carcinoma de Células Acinosas e Adenocarcinoma.

${ }^{2}$ No grupo Outros não carcinomas, escontra-se os tipos histológicos: Tumores Filóides, Angiomiossarcoma, Hemangiossarcoma, Sarcoma, Doença de Hodgkin, Lipossarcoma e Doença de Paget. 
Quanto à correlação entre faixa etária e estadiamento observou-se que a maioria das pacientes acometidas pela Neoplasia de Mama encontrava-se na faixa etária acima de 45 anos ( $\mathrm{n}=827$, equivalente à $72,16 \%$ ), sendo verificada que as mesmas se encontravam em estadiamentos IIB e IIIA.

Em relação à faixa etária e tipo histológico notou-se que o tipo histológico prevalente foi carcinoma ductal infiltrante, com 87,95\% ( $\mathrm{n}=1008)$, principalmente na faixa etária de 4549 anos, correspondendo a 168 casos (16,66\%), acompanhado de carcinoma lobular, correspondente a $2,26 \%(n=26)$, havendo um maior número de casos de carcinoma lobular em mulheres de 45-54 anos, 30,76\% $(n=8)$ e 65-69 anos, $15,38 \%(n=4)$.

Foi constatado que os anos com o maior número de diagnósticos de Neoplasia de Mama foram os anos de 2008, correspondendo a 10,29\% ( $n=118)$, e 2012 correspondendo a 10,20\% ( $\mathrm{n}=117)$ em comparação a todos os anos da pesquisa. No ano de 2008 foram diagnosticadas 23 pacientes no estádio IIA $(19,49 \%)$ e 22 pacientes no estádio IIB $(18,64 \%)$. No ano de 2012 encontrou-se 21 pacientes no estádio IIB (17,94\%) e 21 pacientes no estádio IIIB $(17,94 \%)$. Foi observado com o passar dos anos um aumento no número de diagnóstico de pacientes em todos os estádios.

Em 2000 observamos uma incidência de pacientes no estádio IV, ocorrendo um aumento entre os anos de 2002 à 2012, com uma discreta queda desde então.

Foi averiguado também que os estádios IIA, IIB e IIIA foram os mais incidentes entre 2000 e 2015, com pico diagnóstico de IIA em 2008 e 2011. Com isso observamos que a maioria das pacientes são diagnosticadas com estadiamento clínico avançado. As categorias IIB e IIIA apresentam picos variados no decorrer dos anos de 2000 a 2015.

Em 2015 foi verificado um número abaixo do esperado em comparação com os demais anos, nos levando a questionar sua veracidade. Acreditamos que os dados referentes a esse ano ainda não foram divulgados. 
DESAFIOS: Revista Interdisciplinar da Universidade Federal do Tocantins - V. 2 - n. 02. p.283-306, jan/jun. 2016.

DOI: http://dx.doi.org/10.20873/uft.2359-3652.2016v2n2p283

Tabela 4- Distribuição dos 1146 casos registrados no RHC do HRRA-TO de pacientes com neoplasia de mama correlacionando faixa etária de acometimento com estadiamento.

Table 4-Distribution of 1146 cases recorded in the RHC HRRA-TO of patients with breast cancer correlating age of involvement with staging.

\begin{tabular}{|c|c|c|c|c|c|c|c|c|c|c|c|c|c|c|c|c|c|c|c|}
\hline \multirow{3}{*}{$\begin{array}{l}\text { FAIXA } \\
\text { ETÁRIA }\end{array}$} & \multicolumn{19}{|c|}{ ESTADIAMENTO } \\
\hline & \multicolumn{2}{|c|}{0} & \multicolumn{2}{|c|}{ I } & \multicolumn{2}{|c|}{ II A } & \multicolumn{2}{|c|}{ II B } & \multicolumn{2}{|c|}{ III A } & \multicolumn{2}{|c|}{ III B } & \multicolumn{2}{|c|}{ III C } & \multicolumn{2}{|c|}{ IV } & \multicolumn{2}{|c|}{$\begin{array}{c}\text { SEM } \\
\text { ESTADIAMENTO }\end{array}$} & \multirow[t]{2}{*}{ TOTAL } \\
\hline & $\mathbf{n}$ & $\%$ & $\mathbf{N}$ & $\%$ & $\mathbf{N}$ & $\%$ & $\mathbf{n}$ & $\%$ & $\mathbf{N}$ & $\%$ & $\mathbf{n}$ & $\%$ & $\mathbf{n}$ & $\%$ & $\mathbf{n}$ & $\%$ & $\mathrm{n}$ & $\%$ & \\
\hline $15-24$ & 0 & 0 & 0 & 0 & 1 & 0,08 & 1 & 0,08 & 2 & 0,17 & 0 & 0 & 0 & 0 & 0 & 0 & 2 & 0,17 & 6 \\
\hline $25-34$ & 2 & 0,17 & 3 & 0,26 & 15 & 1,30 & 5 & 0,43 & 12 & 1,04 & 8 & 0,69 & 11 & 0,95 & 9 & 0,78 & 2 & 0,17 & 67 \\
\hline $35-44$ & 3 & 0,26 & 28 & 2,44 & 32 & 2,79 & 45 & 3,92 & 58 & 5,06 & 21 & 1,83 & 11 & 0,95 & 39 & 3,40 & 9 & 0,78 & 246 \\
\hline$>45$ & 9 & 0,78 & 96 & 8,37 & 139 & 12,12 & 154 & 13,43 & 132 & 11,51 & 109 & 9,51 & 49 & 4,27 & 111 & 9,68 & 28 & 2,44 & 827 \\
\hline TOTAL & 14 & & 127 & & 187 & & 205 & & 204 & & 138 & & 71 & & 159 & & 41 & & 1146 \\
\hline
\end{tabular}


DESAFIOS: Revista Interdisciplinar da Universidade Federal do Tocantins - V. 2 - n. 02. p.283-306, jan/jun. 2016.

DOI: http://dx.doi.org/10.20873/uft.2359-3652.2016v2n2p283

\section{Tabela 5 - Distribuição dos 1146 casos registrados no RHC do HRRA-TO de pacientes com neoplasia de mama correlacionando faixa etária de} acometimento com tipo histológico.

Table 5 - Distribution of the 1146 cases recorded in the RHC HRRA-TO of patients with breast cancer correlating age of involvement with histological type.

\begin{tabular}{|c|c|c|c|c|c|c|c|c|c|c|c|c|c|c|c|c|c|}
\hline \multirow{3}{*}{$\begin{array}{l}\text { FAIXA } \\
\text { ETÁRIA }\end{array}$} & \multicolumn{17}{|c|}{ TIPO HISTOLÓGICO } \\
\hline & \multicolumn{2}{|c|}{$\begin{array}{l}\text { Carcinoma } \\
\text { Ductal } \\
\text { Infiltrante }\end{array}$} & \multicolumn{2}{|c|}{$\begin{array}{l}\text { Carcinoma } \\
\text { Lobular }\end{array}$} & \multicolumn{2}{|c|}{$\begin{array}{l}\text { Carcinoma } \\
\text { Ductal não } \\
\text { Infiltrante }\end{array}$} & \multicolumn{2}{|c|}{$\begin{array}{l}\text { Adenocarcinoma } \\
\text { Mucinoso }\end{array}$} & \multicolumn{2}{|c|}{$\begin{array}{l}\text { Carcinoma } \\
\text { Papilífero }\end{array}$} & \multicolumn{2}{|c|}{$\begin{array}{l}\text { Carcinoma } \\
\text { Ductal } \\
\text { In Situ }\end{array}$} & \multicolumn{2}{|c|}{$\begin{array}{l}\text { Outros } \\
\text { carcinomas }\end{array}$} & \multicolumn{2}{|c|}{$\begin{array}{ll}\begin{array}{l}\text { Outros } \\
\text { carcinomas }\end{array} & \text { não }\end{array}$} & \multirow[t]{2}{*}{ TOTAL } \\
\hline & $\mathbf{n}$ & $\%$ & $\mathbf{n}$ & $\%$ & $\mathbf{N}$ & $\%$ & $\mathbf{n}$ & $\%$ & $\mathbf{n}$ & $\%$ & $\mathbf{N}$ & $\%$ & $\mathbf{n}$ & $\%$ & $\mathbf{n}$ & $\%$ & \\
\hline $15-19$ & 2 & 0,17 & 0 & 0 & 0 & 0 & 0 & 0 & 0 & 0 & 0 & 0 & 0 & 0 & 0 & 0 & 2 \\
\hline $20-24$ & 1 & 0,08 & 0 & 0 & 0 & 0 & 0 & 0 & 0 & 0 & 0 & 0 & 0 & 0 & 3 & 0,26 & 4 \\
\hline $25-29$ & 17 & 1,48 & 1 & 0,08 & 0 & 0 & 0 & 0 & 0 & 0 & 0 & 0 & 1 & 0,08 & 1 & 0,08 & 20 \\
\hline $30-34$ & 42 & 3,66 & 0 & 0 & 0 & 0 & 0 & 0 & 0 & 0 & 0 & 0 & 0 & 0 & 2 & 0,17 & 44 \\
\hline 35-39 & 84 & 7,32 & 3 & 0,26 & 2 & 0,17 & 0 & 0 & 0 & 0 & 2 & 0,17 & 4 & 0,34 & 1 & 0,08 & 96 \\
\hline $40-44$ & 131 & 11,43 & 3 & 0,26 & 3 & 0,26 & 1 & 0,08 & 0 & 0 & 0 & 0 & 8 & 0,69 & 2 & 0,17 & 148 \\
\hline $45-49$ & 168 & 14,65 & 4 & 0,34 & 3 & 0,26 & 1 & 0,08 & 1 & 0,08 & 2 & 0,17 & 5 & 0,43 & 2 & 0,17 & 186 \\
\hline $50-54$ & 149 & 13,00 & 4 & 0,34 & 0 & 0 & 2 & 0,17 & 4 & 0,34 & 0 & 0 & 13 & 1,13 & 0 & 0 & 172 \\
\hline $55-59$ & 112 & 9,77 & 1 & 0,08 & 1 & 0,08 & 0 & 0 & 6 & 0,52 & 0 & 0 & 4 & 0,34 & 2 & 0,17 & 126 \\
\hline $60-64$ & 111 & 9,68 & 2 & 0,17 & 0 & 0 & 2 & 0,17 & 0 & 0 & 1 & 0,08 & 6 & 0,52 & 1 & 0,08 & 123 \\
\hline $65-69$ & 79 & 6,89 & 4 & 0,34 & 1 & 0,08 & 2 & 0,17 & 0 & 0 & 0 & 0 & 4 & 0,34 & 1 & 0,08 & 91 \\
\hline $70-74$ & 53 & 4,62 & 1 & 0,08 & 0 & 0 & 0 & 0 & 0 & 0 & 0 & 0 & 7 & 0,61 & 0 & 0 & 61 \\
\hline $75-79$ & 37 & 3,22 & 3 & 0,26 & 0 & 0 & 1 & 0,08 & 0 & 0 & 0 & 0 & 1 & 0,08 & 0 & 0 & 42 \\
\hline $80-84$ & 17 & 1,48 & 0 & 0 & 0 & 0 & 0 & 0 & 0 & 0 & 0 & 0 & 5 & 0,43 & 1 & 0,08 & 23 \\
\hline$>85$ & 5 & 0,43 & 0 & 0 & 0 & 0 & 0 & 0 & 0 & 0 & 0 & 0 & 3 & 0,26 & 0 & 0 & 8 \\
\hline TOTAL & 1008 & & 26 & & 10 & & 9 & & 11 & & 5 & & 61 & & 16 & & 1146 \\
\hline
\end{tabular}


Tabela 6 - Distribuição dos 1146 casos registrados no RHC do HRRA-TO de pacientes com neoplasia de mama correlacionando ano do diagnóstico com estadiamento.

Table 6 - Distribution of 1146 cases reported in the RHC HRRA-TO of patients with breast cancer correlating year of diagnosis with staging. ESTADIAMENTO

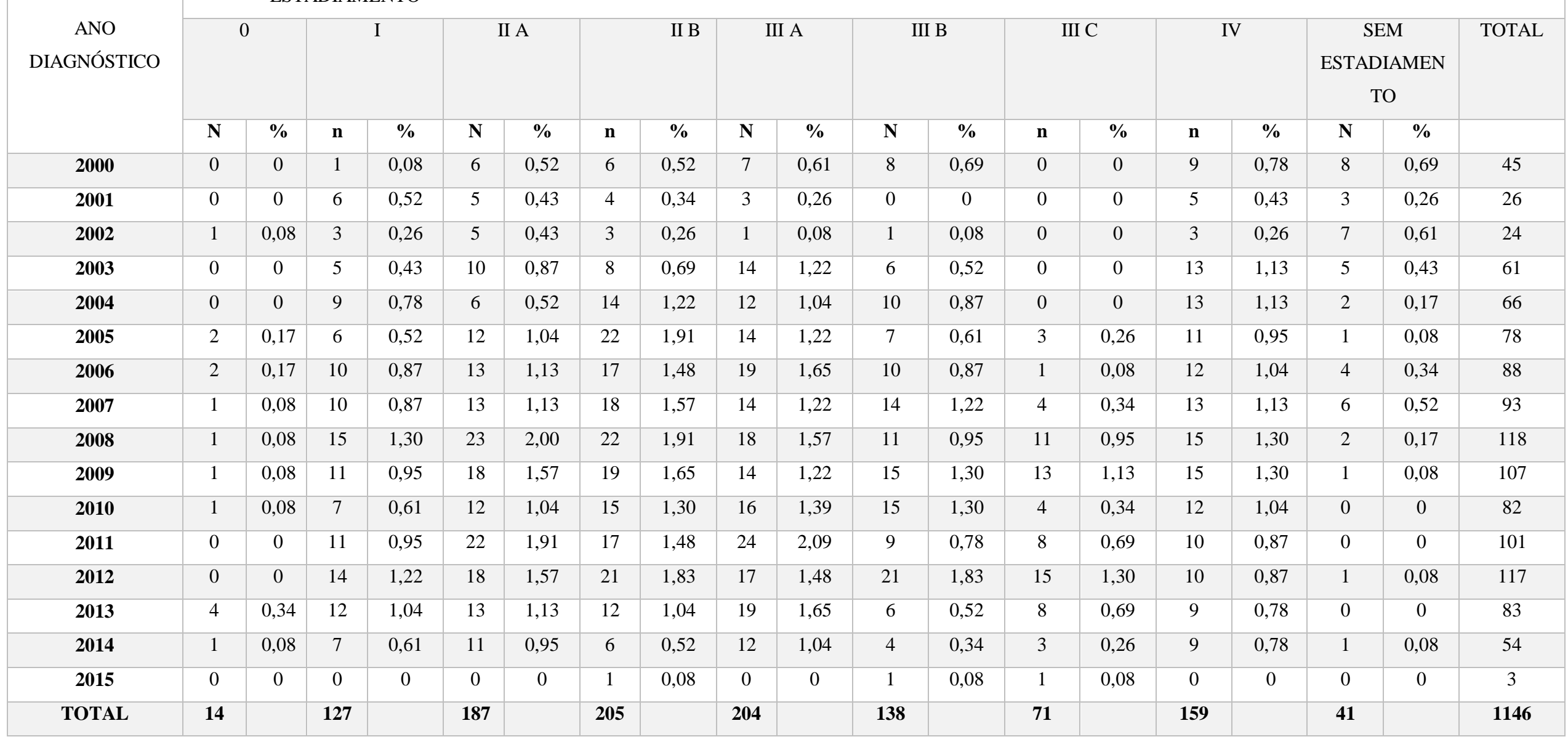




\section{Gráfico 1- Distribuição dos 1146 casos registrados no RHC do HRRA-TO de pacientes com Neoplasia de Mama correlacionando ano do diagnóstico com estadiamento.}

Graphic 1. Distribution of 1146 cases recorded in the RHC HRRA-TO of patients with Breast Neoplasia correlating year of diagnosis with staging.

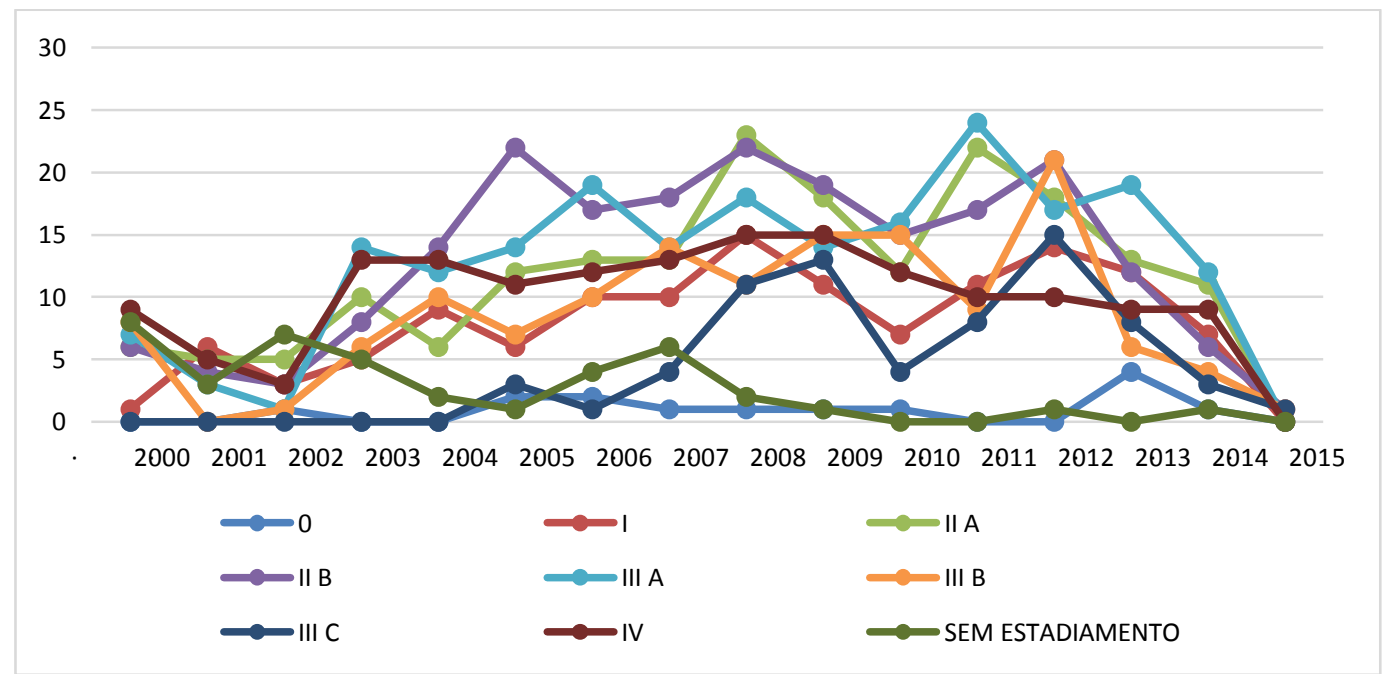

\section{DISCUSSÃO}

A epidemiologia é uma ciência que estuda os determinantes e as condições de ocorrência de doenças e agravos à saúde em populações humanas, utilizando os mais diversos métodos e técnicas. No Brasil, essa disciplina tem vivido um grande avanço nos últimos anos, tanto em sua forma de atuação, como no contingente de profissionais que a ela tem se dedicado. Quantificar ou medir a frequência com que os problemas de saúde ocorrem em populações humanas tem sido alguns dos objetivos dessa área em expansão (CARVALHO, 2003).

Os indicadores de saúde estão inseridos dentro do estudo da epidemiologia com o objetivo de comparar um indivíduo ou uma população, de modo a auxiliar a tomada de decisões racionais, com fundamentos sólidos, ações a recomendar ou aplicar de imediato dentro de um problema de saúde pública. Uma das formas de expressão de seus resultados são os cálculos de frequências (absoluta e relativa) que tem o objetivo de ajudar uma análise posterior de coeficientes e índices de saúde (PEREIRA, 2000).

No presente estudo utilizou-se desses cálculos para avaliação e análise dos dados referentes ao perfil Clínico-Epidemiológico de Mulheres com Neoplasia de Mama atendidas no Hospital Regional de Referência no município de Araguaína-TO . 
O Câncer de Mama é o mais incidente em mulheres, excetuando-se os casos de pele não melanoma. É a quinta causa de morte por câncer em geral (522.000 óbitos) e a causa mais frequente de morte por câncer em mulheres (INCA, 2015).

No Brasil, a Neoplasia de Mama também é a mais incidente (excluídos os tumores de pele não melanoma) em mulheres de todas as regiões, exceto na região Norte, onde o câncer do colo do útero ocupa a primeira posição (INCA, 2015).

Para o ano de 2014 foram estimados 57.120 casos novos, que representam uma taxa de incidência de 56,1 casos por 100.000 mulheres. Para o Tocantins foi estimada uma taxa para o ano de 2014 de 27,58 casos para cada 100 mil mulheres (INCA, 2015).

Apesar da etiologia ainda obscura, o Câncer de Mama possui alguns fatores que tendem a aumentar o risco de apresentação de tal neoplasia, dentre os quais devem ser citados sexo feminino, idade >40 anos, antecedente pessoal de câncer de mama, história familiar (parente de primeiro grau, mãe ou irmã), nuliparidade, primeiro parto após os 30 anos, raça branca, dentre vários outros fatores (MENKE, 2006).

Menos de 1\% de todos os Cânceres de Mama ocorrem em mulheres com menos de 25 anos de idade. A grande maioria tem sua incidência aumentada após os 30 anos, apresentando um platô entre 45 e 50 anos, aumentando progressivamente (CHOY, 2014).

Em nosso estudo podemos observar que a grande maioria dos casos de Neoplasia de Mama ocorreram em mulheres com idade entre 45-49 anos, o equivalente à 16,14\% ( $\mathrm{n}=185)$, já em mulheres com menos de 25 anos esse dado corresponde a 0,52\% (n=6), mostrando uma correlação com os dados da literatura.

Em relação à raça/cor observou-se que 73,21\% (n=839) das mulheres avaliadas eram pardas, não equivalendo aos dados encontrados na literatura, porém sendo o esperado em nosso país já que o mesmo possui uma vasta miscigenação racial.

As medidas preventivas para detecção precoce de Câncer de Mama podem ser influenciadas pelo nível de escolaridade da mulher. Em um estudo realizado em 2013 através do Registro Hospitalar do Câncer(RHC) comparando as mulheres de todas as regiões do país, $(n=12.689)$ entre os anos de 2000 a 2009, contatou-se que a maioria dessas mulheres apresentavam ensino médio completo (PINHEIRO, 2013).

Tal estudo não condiz com nossa pesquisa, em que 43,80\% ( $n=502)$ das mulheres apresentavam nível fundamental incompleto.

O Câncer de Mama sem associação com o fator hereditário é responsável por mais de 90\% dos casos de Câncer de Mama em todo mundo. Dados clínicos, epidemiológicos e 
experimentais demonstram elevado risco de desenvolvimento de Câncer de Mama esporádico relacionado à produção de esteroides sexuais. Condições endócrinas moduladas pela função ovariana, como a menarca precoce, menopausa tardia e gestação, assim como a utilização de estrógenos exógenos, são componentes relevantes do risco de desenvolvimento de Neoplasia Mamária (TIEZZI, 2009).

Em nosso estudo verificou-se que 45,20\% ( $\mathrm{n}=518)$ do total de mulheres avaliadas possuíam história familiar de neoplasia, contra 40,40\% (n=463) que não possuíam essa relação. No entanto, 14,39\% ( $\mathrm{n}=165)$ dessa população foi referenciada como sem informação, não permitindo uma conclusão satisfatória a respeito da influência do fator hereditário nas mulheres avaliadas nesse período.

Em confluência com fatores hormonais, estudos observacionais tem indicado que o comportamento humano relacionado ao estilo de vida, incluindo modificações na dieta e na atividade física, podem aumentar a incidência do Câncer de Mama mundialmente (TIEZZI, 2009).

Alguns fatores bem estabelecidos incluem ingestão de bebida alcoólica, sobrepeso, obesidade na pós-menopausa e exposição à radiação ionizante. O tabagismo foi reconhecido como agente carcinogênico com limitada evidência de aumento do risco de Câncer de Mama em humanos (INCA, 2015).

Avaliamos algumas características relacionadas ao estilo de vida das mulheres estudadas tais como histórico de consumo de tabaco e histórico de consumo de bebida, de forma a buscar uma correlação entre esses fatores e o aumento da incidência de Neoplasia de Mama.

Dentre os 1146 casos de Neoplasia de Mama analisados 35,59\% (n=408) eram fumantes ou já haviam fumado, e 54,10 (n=620) não eram tabagistas.

Em relação ao consumo de bebidas verificou-se que 21,82\% (n=251) consumiam ou já haviam sido consumidores de bebidas alcoólicas, enquanto 67,10\% (n=769) referiam não consumirem ou já terem consumido.

O papel do consumo de álcool no desenvolvimento e progressão do Câncer de Mama gera grande interesse público e debate científico. O mecanismo exato ainda deve ser elucidado, porém, sabe-se que o álcool pode levar a lesão tecidual progressiva através da formação de acetaldeído (BRENNER, 2016).

A presença de acetaldeído e o metabolismo ou a excreção ineficiente do etanol, resulta na formação de espécies reativas de oxigênio, especialmente os superóxido, que afeta a 
carcinogênese através de uma resposta inflamatória. Porém existe a necessidade de mais estudos para estabelecer uma correlação mais fidedigna (SCOCCIANTI, 2014; BRENNER, 2016).

O Câncer de Mama faz parte de um grupo heterogêneo de doenças, com comportamentos diferentes. O espectro de anormalidades proliferativas nos lóbulos e ductos da mama inclui hiperplasia, hiperplasia atípica, carcinoma in situ e carcinoma invasivo. Dentre esses últimos, o carcinoma ductal infiltrante é o tipo histológico mais comum e compreende entre 80 e $90 \%$ do total de casos (INCA, 2015).

Neste estudo verificou-se que 87,95\% $(\mathrm{n}=1008)$ das mulheres avaliadas apresentaram como tipo histológico o carcinoma ductal infiltrante e 2,26\% (n=26) tiveram como tipo histológico o carcinoma lobular. Contudo, os dados referentes à percentagem do carcinoma lobular não está em concordância com a literatura, pois, esses carcinomas são responsáveis por cerca de 10 a 15\% dos Cânceres de Mama invasivos (MARTINS, 2009).

Em um estudo realizado com 12.689 mulheres jovens (18 a 39 anos) em todas as regiões do país, verificou-se que o tipo histológico predominante também foi o carcinoma ductal infiltrante (PINHEIRO, 2013).

Através do sistema TNM o estadiamento clínico é determinado. Este sistema é constituído pelo tamanho do tumor (T), número de linfonodos comprometidos $(\mathrm{N})$ e a presença ou não de metástases (M). O carcinoma in situ é classificado como estádio 0 e os estádios I e II, em geral, referem-se a tumores localizados no órgão de origem. O estádio III refere-se aos tumores com disseminação local extensa, especialmente para linfonodos regionais e os tumores com metástases à distância, classificados como estádio IV (apud PINHEIRO, 2013).

Mulheres entre 50 e 60 anos de idade, estão no grupo com maior incidência de Câncer de Mama. Nesta faixa etária, essa neoplasia apresenta características moleculares de melhor prognóstico, as quais possibilitam mais sucesso nos tratamentos. Além do que, estas mulheres possuem cobertura por programas de rastreamento como mamografia, permitindo diagnósticos em estádios precoces (apud STIVAL, 2014).

Aproximadamente 6,6\% dos de Cânceres de Mama tem sido diagnosticados entre mulheres de 40 anos de idade e mulheres mais jovens. (BRENNER, 2016). Nesta faixa etária a doença apresenta-se de forma mais agressiva, com maior incidência de recorrência, menor sobrevida livre da doença e sobrevida global. Neste grupo de pacientes os tumores, em geral, estão associados a receptores hormonais (RH) negativos, à superexpressão de receptor do 
fator de crescimento epidérmico humano 2 (HER2) e aos estádios de diagnóstico mais avançados em comparação com outras faixas etárias (GARICOCHEA, 2009).

Em um estudo transversal realizado no Brasil, as mulheres com Neoplasia Mamária muito jovens (<35 anos), quando confrontadas àquelas com 35 anos ou mais, demonstraram com maior frequência a presença de tumores com tamanho $>2 \mathrm{~cm}$, status dos linfonodos positivo, presença de metástase, estadiamento clínico avançado ( $\geq$ IIB) e ausência de resposta terapêutica (PINHEIRO, 2013).

Em nosso estudo foi observado que as mulheres com menos de 39 anos ( $n=169$ casos), correspondem a $14,74 \%$ do total de casos $(n=1146)$. Este valor aumenta quando consideramos as mulheres diagnosticadas com Neoplasia de Mama na faixa etária entre 40 e 49 anos ( $\mathrm{n}=335)$, atingindo uma porcentagem de 29,23\% dos casos. As mulheres na faixa etária acima de 50 anos correspondem a 56,02\% (n=642). Esses valores verificados na faixa etária abaixo de 40 anos representam mais que o dobro de mulheres nessa faixa etária com diagnóstico de Câncer de Mama, comparados aos estudos citados. Com estes dados, poderíamos questionar: Onde está o problema? essa percentagem de mulheres jovens com Câncer de Mama é expressiva? Dificuldade de estabelecimento de medidas preventivas para neoplasia nessas mulheres?

Achado semelhante foi descrito em Goiânia em um estudo descritivo e retrospectivo a partir da base de dados do Registro de Câncer de Base Populacional de Goiânia no período de 1989 a 2003. Nesta pesquisa foram coletados 3.204 casos de Câncer de Mama. Sobre a idade das pacientes $15,2 \%$ das mulheres tinham 40 anos ou menos, $27,8 \%$ entre 40 e 50 anos e $57 \%$ tinham mais de 50 anos de idade (MARTINS, 2009).

Contudo, para uma possível elucidação desse problema mais estudos necessitam ser realizados, com intuito de melhoria da assistência à saúde de mulheres jovens com Câncer de Mama, considerando o desfecho clínico desfavorável nessa faixa etária.

A detecção do Câncer de Mama ainda em estádios não invasivos (carcinoma in situ) reduz a frequência de desenvolvimento de câncer de mama invasivo, e aumenta a identificação em estádios iniciais (TNM estádio I), proporcionando assim um tratamento com menor morbidade e chance de cura maior que 90\%. Apesar de os primeiros resultados dos programas sobre a mortalidade levarem cerca de 10 anos para serem notados, a efetividade destes pode ser avaliada indiretamente com a análise do estádio do Câncer de Mama no momento do diagnóstico (MACCHETTI, 2007). 
Em relação ao ano do diagnóstico e o estadiamento, observou-se que no primeiro quinquênio a frequência absoluta dos casos de carcinoma in situ foi de 01(um) caso. Já para os tumores metastáticos (estádio IV) foram 43 casos. A partir de 2002 ocorreu uma elevação evidente no número de casos de todas as categorias do estadiamento TNM, com consequente queda dos casos "sem estadiamento".

Em 2002 foi implantado quatro Centros de Alta Complexidade em Oncologia (CACON) nos estados do Rio de Janeiro, Tocantins e Rio Grande do Sul, promovendo um aumento da cobertura nessas regiões. Esta ação em saúde proporcionou os diagnósticos de casos de Câncer de Mama em Araguaína e região, onde a assistência oncológica necessitava de recursos para melhoria e detecção precoce dos cânceres (INCA, 2002).

A inauguração dos CACON de Araguaína - TO (Hospital Comunitário de Araguaína) aumentou a cobertura de $0 \%$ para $60 \%$ no Estado do Tocantins (INCA, 2002).

Os estádios que apresentaram maior incidência nesse primeiro quinquênio (figura 2) foram IIB, IIIA e IV, todos com estadiamento clínico avançado. Dentre os três, os tumores metastáticos foram os de maior número com 43 casos.

Gráfico 2- Distribuição dos casos registrados no RHC do HRRA-TO de pacientes com Neoplasia de Mama dos anos de 2000 a 2004, correlacionando Ano do Diagnóstico com Estadiamento. Graphic 2 Distribution of cases reported in the RHC HRRA-TO of patients with neoplasm of Mama the years 2000-2004, correlating diagnosis of the Year with staging.

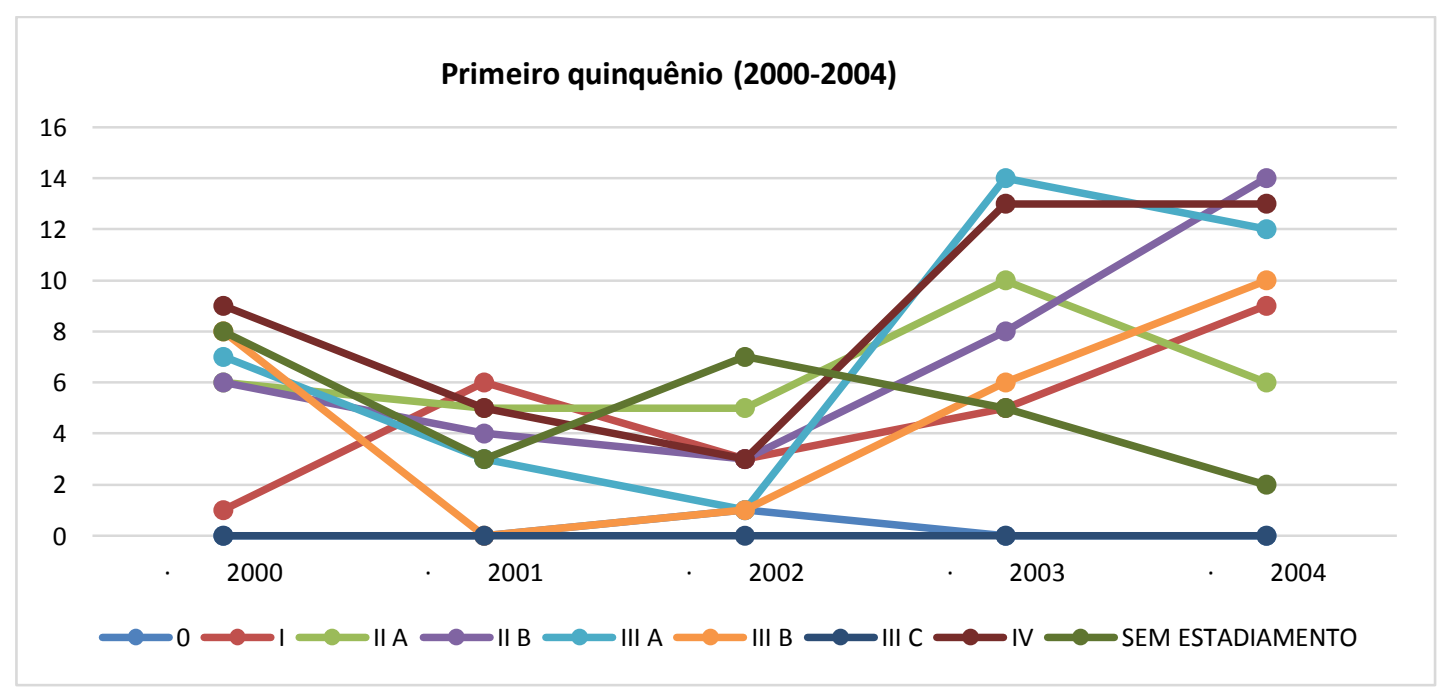

No segundo quinquênio (figura 3) o aumento dos diagnósticos em todas as categorias observados no primeiro gráfico, manteve-se elevado. No estádio IIIC verificou-se aumento de 0 para 32 casos diagnosticados. Ocorreu uma discreta elevação do estádio 0 de 01 caso pra 07 
casos, considerando-se dez anos de pesquisa. Os estádios com maior número de casos desse segundo quinquênio foram IIA, IIB e IIIA, com 79, 98 e 88 casos respectivamente.

Neste gráfico podemos considerar que apesar do crescente número de diagnósticos, ainda observa-se estadimentos clínicos avançados em uma grande parcela da população estudada.

Gráfico 3- Distribuição dos casos registrados no RHC do HRRA-TO de pacientes com Neoplasia de Mama dos anos de 2005 a 2009, correlacionando Ano do Diagnóstico com Estadiamento. Graphic 3 Distribution of cases reported in the RHC HRRA-TO of patients with neoplasm of Mama the years 2005-2009, correlating diagnosis of the Year with staging.

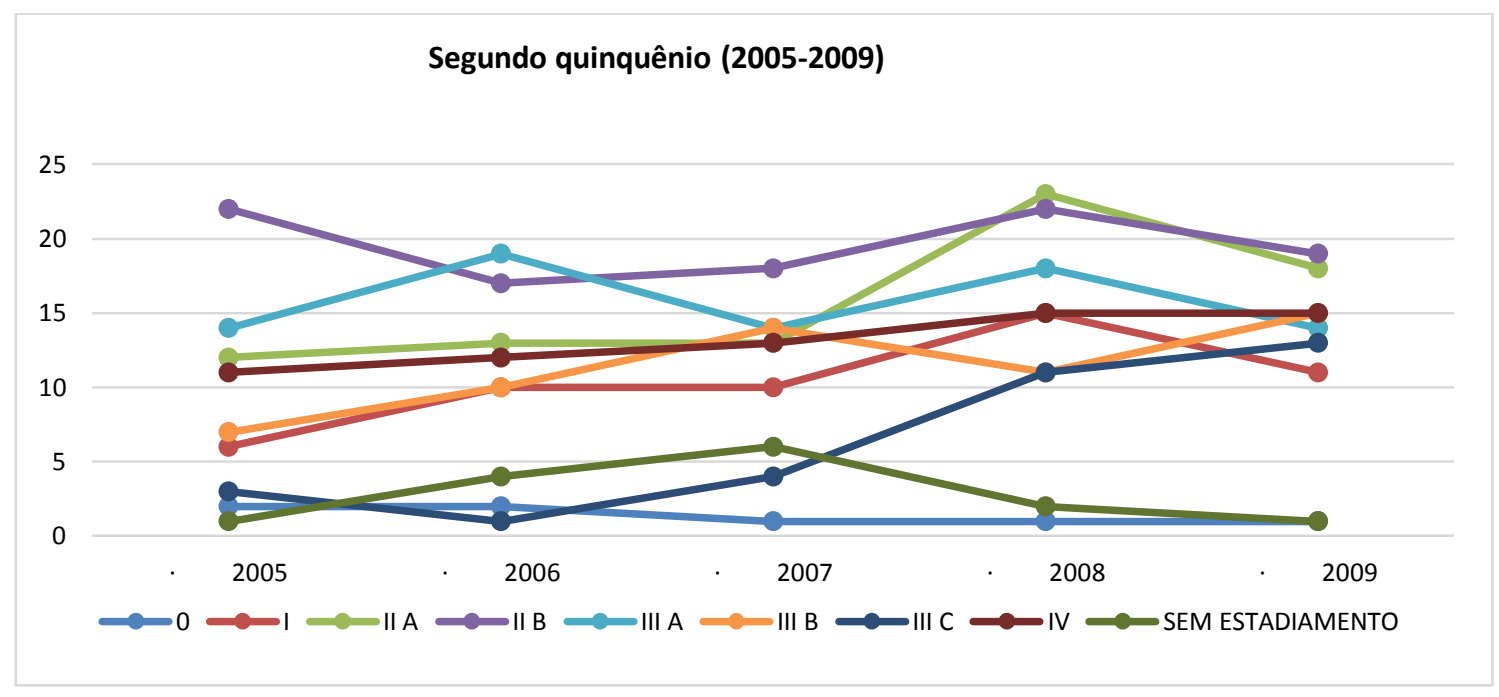

Nota-se no terceiro quinquênio (figura 4) um elevado número de casos no estádio IIIA (n=88). Em comparação ao segundo quinquênio, os casos de câncer diagnosticados como doença metastática sofreu redução de 66 casos para 50 casos. Essa leve redução pode sugerir melhorias que ocorreram no sistema de saúde para a detecção de casos de Câncer de Mama.

Em 2014 verificou-se uma queda expressiva no número de diagnósticos, devendo-se ao fato de melhoria do serviço de oncologia na capital do estado, havendo migração de pacientes.

Os demais dados de outros estádios mantiveram-se semelhante aos do segundo quinquênio. No período estudado, houve redução de 8 registros para 1 registro dos casos sem informação quanto ao estadiamento da doença. 
Gráfico 4- Distribuição dos casos registrados no RHC do HRRA-TO de pacientes com Neoplasia de Mama dos anos de 2010 a 2015, correlacionando Ano do Diagnóstico com Estadiamento. Graphic 4 Distribution of cases reported in the RHC HRRA-TO of patients with neoplasm of Mama the years 2010-2015, correlating diagnosis of the Year with staging.

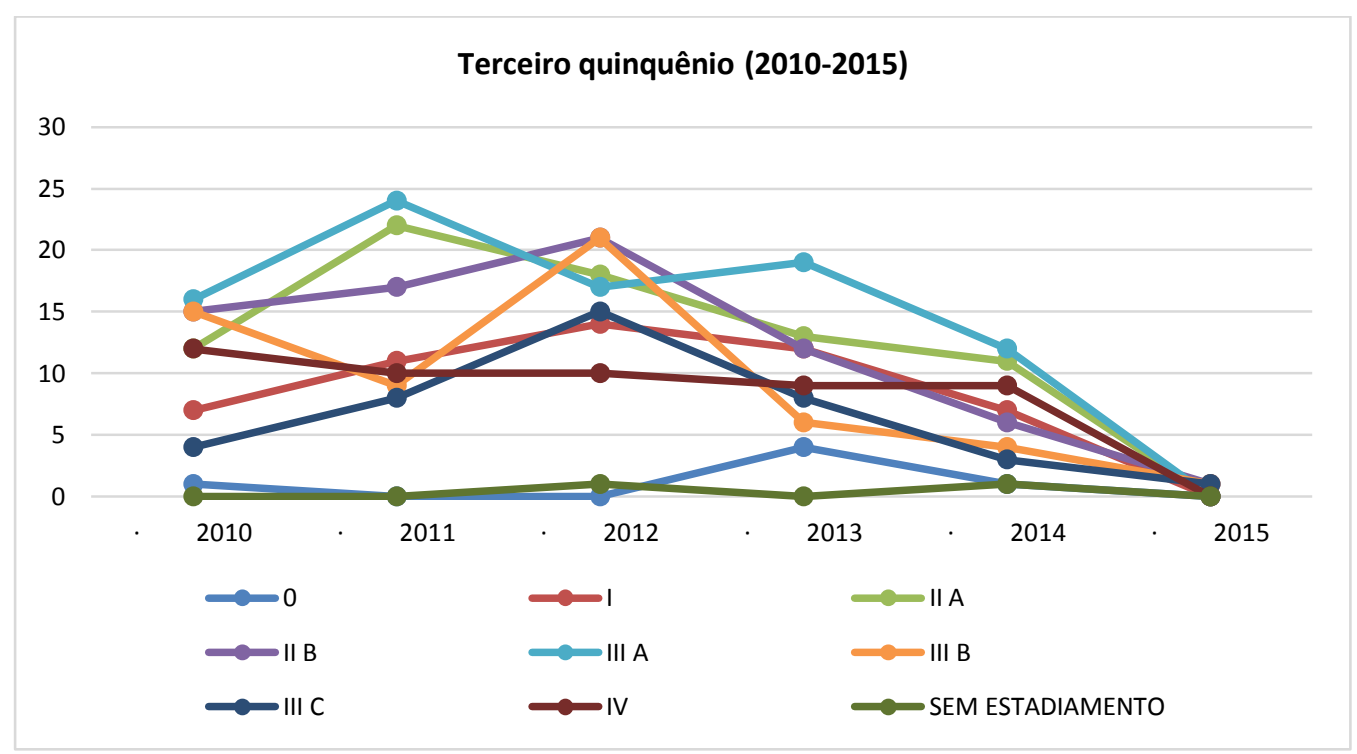

O desenvolvimento de estratégias para diminuir o retardo no diagnóstico e início do tratamento do câncer de mama requer uma ampla compreensão dos fatores associados a esse atraso (PAIVA, 2015).

A sobrevida média após cinco anos na população de países desenvolvidos é aproximadamente $85 \%$. No entanto, em países em desenvolvimento, a sobrevida fica próximo aos 60\%. Provavelmente, essas taxas de mortalidade mantêm-se elevadas devido à doença ainda ser diagnosticada em estádios avançados (PORTO, 2013).

A alta taxa de mortalidade No Brasil pode ser explicada parcialmente pelo fato de, em média, 60\% dos tumores de mama terem seu diagnóstico em estádios avançados (PAIVA, 2015).

Uma das principais dificuldades e fator determinante para o diagnóstico tardio da Neoplasia de Mama é o atraso para a investigação de lesões mamárias suspeitas (PAIVA, 2015).

Os custos hospitalares associados a ausências no trabalho, consequência da recuperação dos doentes em tratamento, acarretam uma diminuição da produtividade pessoal, além de alto impacto social (RODRIGUES, 2010). 


\section{CONSIDERAÇÕES FINAIS}

Diante dos resultados obtidos com a realização do presente estudo sobre as mulheres atendidas no Hospital Regional de Referência de Araguaína com neoplasia de mama no período de 2000 a 2015, podemos concluir que:

1. A Neoplasia de Mama acomete principalmente mulheres pardas, na faixa etária entre 45 e 49 anos, com nível médio de escolaridade;

2. A maioria das mulheres afetadas eram casadas, não consumiam bebidas alcoólicas e não eram tabagistas;

3. As mulheres avaliadas apresentavam como tipo histológico predominante o carcinoma ductal infiltrante. Em relação ao fator hereditário o estudo não possibilitou uma avaliação satisfatória.

4. O carcinoma lobular apresentou um percentual abaixo do encontrado na literatura, levantando hipóteses sobre a autenticidade desse dado (fidedignidade do exame histopatológico?)

5. Uma grande quantidade de mulheres jovens ( $<45$ anos) apresentaram neoplasia de mama, o mesmo correspondendo à estágios mais avançados da doença, nos levando a questionamentos sobre o porque de tais dados. Há dificuldade em acesso ao serviço de saúde? Dificuldade no estabelecimento de medidas preventivas para a neoplasia de mama?

6. Os estádios IIA, IIB e IIIA foram os mais incidentes entre 2000 e 2015, sendo a maioria das pacientes diagnosticadas em estadiamento clínico avançado.

Constatamos então que a assistência de saúde oncológica às mulheres de Araguaína e região necessita de melhorias em todas as suas esferas, desde atenção básica até alta complexidade, envolvendo estrutura física e recursos humanos para tal.

\section{REFERÊNCIAS}

ANDRADE, Simone Aparecida Fernandes de. Câncer de Mama: Um Problema de Saúde Pública. Revista UNILUS Ensino e Pesquisa, São Paulo, v.11 n.23, p. 70-77, 2014.

BRENNER, Darren R. et al. Breast cancer survival among young women: a review of the role of modifiable lifestyle factors. Cancer Causes Control. Canada, v.27, p.459-472, 2016.

CARVALHO, Diana Maul. Epidemiologia - História e Fundamentos. In: MEDRONHO, Roberto A. et al. Epidemiologia. São Paulo: Atheneu, 2003. p.3-13.

CHOY, J.O.; GIULIANO, A.E. Câncer de Mama. BEREK, Jonathan S. Tratado de Ginecologia. 15 ed. Rio de Janeiro: Guanabara Koogan, 2014. 40, p. 1135-1152. 
Controle do câncer de mama- Conceito e magnitude

<http://www2.inca.gov.br/wps/wcm/connect/acoes_programas/site/home/nobrasil/programa controle_cancer_mama/conceito_magnitude> Acesso: em 13/08/15.

GARICOCHEA, Bernardo, et al. Idade como fator prognóstico no câncer de mama em estádio inicial. Rev Saúde Pública. Porto Alegre, v.43, n.2, p.311-317, 2009.

LESTER, Susan C. A Mama. In: KUMAR, Vinay; ABBAS, Abul K.; FAUSTO, Nelson.

Robbins \& Cotran Patologia: Bases patológicas das doenças. $8^{\mathrm{a}}$ ed. Rio de Janeiro: Elsevier, 2010. 23, p.1073-1103.

MACCHETTI, Alexandre Henrique. Estadiamento do Câncer de Mama Diagnosticado no Sistema de Saúde de São Carlos. Revista de Medicina de Ribeirão Preto. Ribeirão Preto (SP), v.40, n.3, p.394-402, 2007.

MARTINS, Edesio, et al. Evolução temporal dos estádios do câncer de mama ao diagnóstico em um registro de base populacional no Brasil Central. Rev Bras Ginecol Obstet. Goiânia (GO), v.31, n.5, p.219-223, 2009.

MENKE, C. Henrique, et al. Câncer de Mama. In: FREITAS, Fernando, et al. Rotinas em Ginecologia. 5aed. Porto Alegre: Artmed, 2006. 37, p. 419-436.

PAIVA, Christiano José Kuhl de; CESSE, Eduarda Ângela Pessoa. Aspectos Relacionados ao Atraso no Diagnóstico e Tratamento do Câncer de Mama em uma Unidade Hospitalar de Pernambuco. Revista Brasileira de Cancerologia. Rio de Janeiro, v.61, n.1, p.23-30, 2015. PEREIRA, Maurício Gomes. Indicadores de Saúde. In: PEREIRA, Maurício Gomes. Epidemiologia Teoria e Prática. Rio de Janeiro: Guanabara Koogan, 2000. 4, p. 49-75. PINHEIRO, Aline Barros, et al. Câncer de Mama em Mulheres Jovens: Análise de 12.689 Casos. Revista Brasileira de Cancerologia. Rio de janeiro, v.59, n.3, p.351-359, 2013. PORTO, Marco Antônio Teixeira; TEIXEIRA, Luiz Antônio; SILVA, Ronaldo Correia Ferreira. Aspectos Históricos do Controle de Câncer de Mama no Brasil. Revista Brasileira de Cancerologia. Rio Janeiro: Fiocruz, v.59, n.3, p.331-339, 2013.

RELATÓRIO ANUAL 2002. Instituto Nacional do Câncer- Ministério da Saúde. Relatório. Rio de Janeiro, 2002.

RODRIGUES, Juliana Stoppa Menezes; FERREIRA, Noeli Marchioro Liston Andrade. Caracterização do perfil epidemiológico do câncer em uma cidade do interior paulista: conhecer para intervir. Revista Brasileira de Cancerologia, São Paulo, v. 56, n. 4, p. 431-441, 2010.

SCOCCIANTE, Chiara et al. Female Breast Câncer and Alcohol Consumption: A Review of the Literature. Am J Prev Med. France, Paris, v.46, n.1, p.16-25, 2014.

STIVAL, Rebecca S. Marchesini; PRESTES, Ana Lúcia de Oliveira; MANSANI, Fábio Postiglione. Câncer de mama em mulheres jovens: uma análise do estadiamento clínico inicial e dos subtipos moleculares dos tumores. Rev Bras Mastologia. Ponta Grossa (PR), v. 24, n.1, p.17-22, 2014.

TIEZZI, Daniel Guimarães. Epidemiologia do Câncer de Mama. Rev Bras Ginecol Obstet, v. 31, n.5, p. 213-5, 2009.

\section{Maianna Macêdo de Sousa}

Graduada em Medicina e em Enfermagem ambos pelo Instituto Tocantinense Presidente Antônio Carlos, Faculdade de Ciências Humanas, Econômicas e da Saúde de Araguaína (FAHESA/ITPAC).

E-mail: maiannams@gmail.com

Endereço: Instituto Tocantinense Presidente Antônio Carlos - Curso de Medicina - Av. Filadélfia, 568 - St. Oeste, Araguaína - TO, 77816-540 


\section{Sabrina Barreto Figueredo}

Graduada em Medicina pelo Instituto Tocantinense Presidente Antônio Carlos, Faculdade de Ciências Humanas, Econômicas e da Saúde de Araguaína (FAHESA/ITPAC).

E-mail: sabrinabfigueredo@gmail.com

Endereço: Instituto Tocantinense Presidente Antônio Carlos - Curso de Medicina - Av. Filadélfia, 568 - St. Oeste, Araguaína - TO, 77816-540

\section{Reinaldo Magalhães Fernandes}

Professor do Instituto Tocantinense Presidente Antônio Carlos, médico mastologista do Governo do Estado de Tocantins, médico mastologista da Associação dos Voluntários e Amigos dos Portadores de Câncer em Araguaína e médico mastologista do Hospital e Maternidade Dom Orione.

E-mail: reinaldomf@globo.com

Endereço: Instituto Tocantinense Presidente Antônio Carlos - Curso de Medicina - Av. Filadélfia, 568 - St. Oeste, Araguaína - TO, 77816-540 